\title{
ORIGINAL ARTICLE \\ Morbidity of urinary tract infection after urodynamic examination of hospitalized SCI patients: the impact of bladder management
}

\author{
R Böthig ${ }^{1}$, K Fiebag ${ }^{1}$, R Thietje ${ }^{2}$, M Faschingbauer $^{3}$ and S Hirschfeld ${ }^{2}$
}

\begin{abstract}
Study design: Non-interventional, descriptive-observational cohorts study.
Objectives: To assess the incidence of urinary tract infection (UTI) after urodynamic examination in patients with spinal cord injury (SCl) according to bladder management.
\end{abstract}

Setting: Level 1 trauma center

Methods: Between January and December 2010 a total of 133 consecutive, hospitalized SCI patients were included and classified according to their bladder management. Urine specimen was obtained at the beginning of the urodynamic studies (UDS) and 3-5 days thereafter. 'Significant bacteriuria' (SBU) is defined by a CBU per $\mathrm{ml}$ level $\geqslant 10^{5}$ in a urine culture. UTI thus is defined as a combination of a SBU and $\geqslant 100$ leukocytes per $\mu \mathrm{l}$ in urine analysis.

Results: The overall incidence of UTI post UDS was $15.79 \%$. In patients with sterile urine prior to urodynamics UTI was ascertained in $8.6 \%$ (de-novo-UTI). In contrast, $32.5 \%$ of the patients with SBU prior to UDS showed UTI 3 days later. There were only minor differences in the incidence of de-novo-UTIs in $\mathrm{SCl}$ patients who emptied their bladder by intermittent self catheterization or intermittent catheterization by attendant $(8.82 \%$ and $6.67 \%$, respectively). In SCI patients with reflex voiding however, the frequency of de-novo-UTIs was twice as high (14.28\%).

Conclusion: The recommendation of antibiotic prophylaxis for all SCI patients undergoing urodynamic examination is not commonly accepted and according to our data not justified. However, the analysis of subgroups revealed that SCI patients with unsuspected SBU prior to UDS and patients with reflex voiding are possibly at higher risk to acquire post-UDS infection.

Spinal Cord (2013) 51, 70-73; doi:10.1038/sc.2012.107; published online 11 September 2012

Keywords: urodynamics; spinal cord injury; urinary tract infection; significant bacteriuria; antibiotic prophylaxis

\section{INTRODUCTION}

Patients with spinal cord injury (SCI) frequently suffer from neurogenic lower urinary tract dysfunction (NLUTD). Especially during their initial rehabilitation period, repeated urodynamic studies (UDS) have to be conducted in order to ensure low storage pressure in the bladder and thus to enable an adequate voiding pattern. During the subsequent lifelong follow-up care they need re-evaluation of their bladder situation in hazard-adapted intervals. The crucial investigation again is a urodynamic examination. ${ }^{1}$

SCI-patients with NLUTD have an increased risk for urinary tract infection (UTI). This is due to the paralyzed bladder with detrusor over- or underactivity and the emptying by intermittent catheterization (IC) or reflex voiding., ${ }^{2,3}$

There is also a low, but well-known risk for UTI and a moderate risk for bacteriuria caused by UDS, in patients with non-NLUTD. ${ }^{4}$

As the incidence of UTI's after urodynamic testing in patients with NLUTD seems to be scarcely investigated, only a small amount of adequate data exists. Therefore we analyzed the incidence of UTI's after urodynamic investigation in SCI patients, especially regarding the different voiding methods.

\section{MATERIALS AND METHODS}

Between January and December 2010 a total of 210 SCI patients scheduled to our neuro-urological department for UDS were included in the prospective observational study. All patients were hospitalized for primary rehabilitation or treatment of complications due to their SCI. 31 out of the 210 patients had an indwelling suprapubic or urethral catheter and further 46 received an antibiotic treatment for various reasons. Therefore they had to be excluded from the study, thus minimizing the feasible population under study to a total of 133 patients.

116 were male with an age interval between 19 to 79 years $($ mean $=45)$ and 17 were female in the age interval of $32-75$ years $($ mean $=60)$. The patients were divided into three specific classes characterized by their specific method of bladder management. (I) Intermittent self catheterization (ISC) was performed by 51 patients, (II) IC by attendant (trained nurse) was provided for 63 patients while the last 19 patients (III) were using triggered reflex voiding. Furthermore the patients were differentiated by whether they had (A) sterile urine (no CBU per $\mathrm{ml}$ ) or (B) unsuspected bacteriuria prior to UDS.

'Significant bacteriuria' (SBU) is defined in our department by a CBU per $\mathrm{ml}$ level $\geqslant 10^{5}$ in a urine culture sample. UTI thus is defined as a composition of a SBU and $\geqslant 100$ leukocytes per $\mu \mathrm{l}$ in a urine analysis according to the definition of the 'Manual of Neuro-Urology and Spinal Cord Lesion'. Annotation: low bacterial counts $\left(10^{2} \mathrm{CBU}-10^{4} \mathrm{CBU}\right.$ per $\left.\mathrm{ml}\right)$ were only seen

${ }^{1}$ Department Neuro-Urology, BG Trauma Hospital Hamburg, Hamburg, Germany; ${ }^{2}$ Centre for Spinal Injuries, BG Trauma Hospital Hamburg, Hamburg, Germany and ${ }^{3}$ Department of Traumatology, Orthopedics and Sports Traumatology, BG Trauma Hospital Hamburg, Hamburg, Germany

Correspondence: Dr R Böthig, Department Neuro-Urology, BG Trauma Hospital Hamburg, Bergedorfer Street 10, Hamburg 21033, Germany.

E-mail: r.boethig@buk-hamburg.de

Received 29 September 2011; revised 23 July 2012; accepted 31 July 2012; published online 11 September 2012 
in some of the patients treated with antibiotics prior to UDS and all antibiotictreated patients were excluded from the study as mentioned above.

Urodynamic studies were performed according to the ICS standards and good urodynamic practice ${ }^{6}$ with a sterile catheterization technique. The patients received no antibiotic prophylaxis. All 133 patients provided a urine specimen for urine analysis and urine culture at the start of the urodynamic examination and the comparison specimen 3-5 days later.

\section{RESULTS}

All patients

With the results of the initial samples taken prior to the UDS, the population was divided into two distinctive groups: (A) $93(70.0 \%)$ patients had sterile urine cultures and (B) 40 patients (30.0\%) showed unsuspected SBU (Table 1, Table 2).

The reference samples taken after the UDS showed that out of 93 patients with sterile urine (A), 81 patients $(87.1 \%)$ still had sterile urine, while 4 patients $(4.3 \%)$ did develop SBU (de-novo-SBU) and further 8 patients $(8.6 \%)$ showed an additional leucocyturia $\geqslant 100$ per $\mu \mathrm{l}$ (de-novo-UTI).

Out of the second group (B) with 40 patients showing SBU at the initial presentation, 20 patients $(50.0 \%)$ had sterile urine $3-5$ days after the UDS. Seven patients $(17.5 \%)$ had an ongoing SBU, while the remaining 13 patients $(32.5 \%)$ developed UTI.

According to the overall sample results it is concluded that 101 out of 133 patients (75.94\%) remained without any infection post UDS. In 32 cases $(24.06 \%)$ the urine culture samples revealed significant

Table 1 Urine status at UDS and 3-5 days later according to bladder management

\begin{tabular}{|c|c|c|c|}
\hline \multirow[t]{2}{*}{ At UDS } & \multicolumn{3}{|c|}{ 3-5 Days after UDS } \\
\hline & Sterile & $\geqslant 105$ & $\geqslant 105+\geqslant 100$ per $\mu \mathrm{l}$ \\
\hline \multicolumn{4}{|l|}{ ISC, $n=51$} \\
\hline Sterile, $n=34$ & 30 & 1 & 3 \\
\hline$\geqslant 10^{5}, n=17$ & 10 & 3 & 4 \\
\hline \multicolumn{4}{|c|}{ IC by attendant, $n=63$} \\
\hline Sterile, $n=45$ & 40 & 2 & 3 \\
\hline$\geqslant 10^{5}, n=18$ & 8 & 3 & 7 \\
\hline \multicolumn{4}{|c|}{ Reflex voiding, $n=19$} \\
\hline Sterile, $n=14$ & 11 & 1 & 2 \\
\hline$\geqslant 10^{5}, n=5$ & 2 & 1 & 2 \\
\hline \multicolumn{4}{|l|}{ All, $n=133$} \\
\hline Sterile, $n=93$ & 81 & 4 & 8 \\
\hline$\geqslant 10^{5}, n=40$ & 20 & 7 & 13 \\
\hline
\end{tabular}

Abbreviations: IC, intermittent catheterization; ISC, intermittent self catheterization; UDS, urodynamic studies. bacteriuria (SBU), whereof $11(8.27 \%)$ were without and $21(15.79 \%)$ with a significant leucocyturia level thus proving UTI.

(I) Intermittent self catheterization. Out of 51 patients emptying their bladder with aseptic ISC, 34 had sterile urine before UDS (A) while 17 patients underwent the examination despite SBU (B).

From those with sterile urine, 30 patients $(88.24 \%)$ remained sterile post UDS, 1 patient (2.94\%) developed de-novo-SBU and 3 patients $(8.82 \%)$ suffered from de-novo-UTI.

Out of 17 patients with SBU prior to urodynamic testing, 10 patients showed sterile reference samples, 3 patients developed SBU and the last 4 patients UTI.

In summary, it can be stated that 40 out of 51 ISC patients (78.43\%) did not show signs of either SBU or UTI post UDS according to our criteria. In 4 patients $(7.84 \%)$ a SBU was ascertained while the remaining 7 (13.72\%) developed UTI.

(II) Intermittent catheterization by attendant. The largest patient group (63 patients) provided the following results. Forty-five patients had sterile urine prior to UDS (A) and 18 presented with SBU (B).

Out of the first group (A), 40 patients $(88.89 \%)$ remained sterile urine post UDS. Two patients $(4.44 \%)$ showed de-novo-SBU and three $(6.67 \%)$ de-novo-UTI.

The reference samples of the second group (B) showed that eight patients had ongoing sterile urine post UDS, three patients developed SBU and seven patients UTI, respectively.

Summing up, 48 out of 63 patients $(76.2 \%)$ with IC by attendant remained without SBU or UTI, 5 patients $(7.93 \%)$ developed SBU and 10 patients $(15.87 \%)$ UTI.

(III) Reflex voiding. Nineteen out of the 133 SCI patients under study emptied their bladders by reflex voiding, with or without external collecting devices. Prior UDS 14 had sterile urine (A) while 5 showed unsuspected SBU (B).

Out of 14 patients with sterile urine at urodynamics (A), 11 (78.58\%) showed sterile reference samples post UDS, 1 (7.14\%) developed de-novo-SBU and the remaining 2 (14.28\%) de-novo-UTI.

The reference samples of the five reflex-voiding patients with SBU (B) showed that two (40\%) had sterile samples post UDS, one $(20 \%)$ still had SBU and two (40\%) patients developed UTI.

In summary, the post-UDS reference samples of 19 patients without catheterization showed no infection in 13 patients $(68.43 \%), 2$ patients $(10.52 \%)$ with SBU and the 4 remaining patients (21.05\%) with UTI.

\section{Summary of the results}

The synopsis of the findings presented above shows that $15.79 \%$ of all SCI patients undergoing UDS, regardless of preexisting SBU,

Table 2 post-UDS UTI and UTI + SBU (in percentages) according to bladder management

\begin{tabular}{|c|c|c|c|c|c|c|c|c|}
\hline \multicolumn{3}{|c|}{ All (in \%) } & \multicolumn{3}{|c|}{ Sterile at urodynamic (in \%) } & \multicolumn{3}{|c|}{ SBU at urodynamic (in \%) } \\
\hline & UTI & $U T I+S B U$ & & UTI & $U T I+S B U$ & & UTI & $U T I+S B U$ \\
\hline ISC, $n=51$ & 13.72 & 21.57 & ISC, $n=34$ & 8.82 & 11.76 & ISC, $n=17$ & 23.5 & 41.18 \\
\hline IC by attendant, $n=63$ & 15.87 & 23.81 & IC by attendant, $n=45$ & 6.67 & 11.11 & IC by attendant, $n=18$ & 38.89 & 55.56 \\
\hline Reflex-voiding, $n=19^{a}$ & $21.05^{\mathrm{a}}$ & $31.57^{a}$ & Reflex-voiding, $n=14^{\mathrm{a}}$ & $14.28^{\mathrm{a}}$ & $21.42^{\mathrm{a}}$ & Reflex-voiding, $n=5^{a}$ & $40^{a}$ & $60^{\mathrm{a}}$ \\
\hline All, $n=133$ & 15.79 & 24.06 & all, $n=93$ & 8.6 & 12.9 & all, $n=40$ & 32.5 & 50 \\
\hline
\end{tabular}

Abbreviations: IC, intermittent catheterization; ISC, intermittent self catheterization; SBU, Significant bacteriuria; UDS, urodynamic studies.

aplease note that this group is very small. 
developed a UTI after the urodynamic examination. In patients entering the UDS with sterile urine samples, the incidence of a de-novo-UTI was only $8.6 \%$.

There were only minor differences between patients with ISC and IC by attendant (incidence of de-novo-UTIs, $8.82 \%$ and $6.67 \%$, respectively), but in SCI patients with triggered reflex voiding, who emptied the bladder without regular catheterization, the occurrence of de-novo-UTIs post UDS was almost twice as high (14.28\%). It must be noted that our study group of reflex voiding SCI patients was small (only 19 patients).

Moreover, it has to be pointed out that SCI patients with unsuspected asymptomatic bacteriuria prior to UDS (B), regardless of their bladder emptying method, carried a considerably higher risk to develop UTI $(32.5 \%)$ than the comparison group (A) of patients with sterile urine prior UDS (8.6\%).

\section{DISCUSSION}

Almost all SCI patients suffer from NLUTD and therefore UDS is essential for these patients in order to assess the bladder function during their initial rehabilitation period and the following lifelong surveillance. Although UDS are frequently used, there still exists a lack of clear knowledge about the value and necessity of antibiotic prophylaxis in the high-risk group of SCI patients.

Even if the UDS is performed according to the strict regulations of the International Continence Society ${ }^{5}$ in non-neurogenic patients, there is a distinctive risk to acquire SBU, whereas UTIs due to UDS are infrequent.

The International Continence Society scrutinized the literature concerning complications of urodynamic testing on their fourth 'Consultation on Incontinence' and stated that 'complications are rare but antibiotic prophylaxis can be advocated' (with 'Level of evidence $\left.{ }^{\prime}\right) .^{7}$ In contrast, a systematic literature review revealed that there is rather a moderate to low level of evidence suggesting no need for antibiotic prophylaxis in UDS. However, the authors stated that 'the same low level of evidence may support the use of antibiotic prophylaxis in those patients with known increased risk for infections (for example, neurogenic bladder).8 Another meta-analysis, based on a systematic review of effectiveness and safety of prophylactic antibiotics in urodynamics found eight randomized controlled trials with 995 patients, pointing out a $40 \%$ reduction in the risk of SBU for (non-neurogenic) patients receiving prophylactic antibiotics. The authors concluded that 'one would need to give prophylactic antibiotics to 13 individuals undergoing UDS to prevent one significant bacteriuria of unknown clinical significance. ${ }^{9}$

In a decision analytic model it was calculated that antibiotic prophylaxis in urodynamics is not beneficial unless the occurrence rate of UTI without applied antibiotics exceeds the threshold value of $10 \%{ }^{10}$

Therefore, the recommendations even for patients with nonneurological LUTD are inconsistent, but there exists a certain consensus that an antibiotic prophylaxis is not necessary, unless distinctive risk factors are present.

It is widely accepted that SCI patients with a neurogenic bladder have an increased risk for UTIs, due to various reasons, for example, elevated intravesical pressure, incomplete voiding or the use of catheterization. Unfortunately there is a lack of randomized, prospective studies concerning the problem of UTIs in SCI patients after urodynamics.

\section{'What can we know?'}

In a comprehensive descriptive study ${ }^{11}$ only 2 of 626 patients with NLUTD (530 with SCI, all patients received a single dose gentamycine prior to the procedure) developed a post-UDS UTI (defined as 'clinical symptoms' and $>10^{5} \mathrm{CBU}$ per $\mathrm{ml}$ ). Furthermore, the authors queried institutions within the United States by telephone about their measures to prevent UTI during urodynamics. The results revealed a wide range of protocols, which mostly included an antibiotic treatment directed towards Gram-negative bacteria but in various dosages, frequency and length.

To this day, only one randomized, prospective study on specifically SCI patients is available. ${ }^{12}$ The authors proved an advantage of prophylactic antibiotic treatment, showing that out of a group of 18 patients receiving $500 \mathrm{mg}$ ciprofloxacin orally over 3 days, none developed UTI post UDS, while 3 out of 22 patients in the placebo group did develop UTI. Regrettably the number of participants was too small to reach statistical significance.

A non-randomized, prospective study with 72 SCI patients in an outpatient setting ${ }^{13}$ revealed a post-interventional UTI rate of $9.7 \%$, comparable to our study (8.6\%). But in contrast to our results this study showed no verifiable difference in the incidence of infection in patients with triggered reflex voiding $(n=23)$ compared with those who are performing IC $(n=49)$ (this study did not differentiate between ISC and IC by attendant). Conversely we found an occurrence of $14.28 \%$ post-UDS UTI in patients with reflex emptying and of $7.59 \%$ in patients with IC $(8.82 \%$ in ISC and $6.67 \%$ in IC by attendant). However, it has to be considered that the number of patients with reflex voiding was relatively small in both studies ( $n=23$ and $n=19)$.

Also incongruent were the results with regard to the occurrence of UTI in the patient group revealing asymptomatic bacteriuria prior to the UDS, with no cases in the study mentioned above versus $32.5 \%$ in our analysis. The authors argue that infection prophylaxis is generally justified in SCI patients undergoing UDS, but they did not detect any subgroup that is at a higher risk to acquire UTI during urodynamics.

Our results are also conflicting to an older study, ${ }^{14}$ revealing a threefold increase of UTI's in cervical-level SCI patients who underwent invasive procedures. In our study all of the patients with IC by attendant were tetraplegic and their rate of post-interventional UTI was only slightly higher than in ISC patients (concerning both patient groups: with and without SBU prior to UDS, see Table 2). Accordingly, not the level of injury but the way of bladder management seems to be crucial. Otherwise it has to be considered that this discrepancy may be influenced by the fact that the out-patient population of these study possibly were catheterized by less well trained personal than our in-patient group.

Overall, it can be concluded that the available published data for SCI patients are inconsistent and the evidence is poor. Neither the EAU-guidelines on NLUTD (http://www.uroweb.org/gls/pdf/19 Neurogenic_LR\%20II.pdf) nor the US Consortium for Spinal Cord Medicine $^{15}$ give a general recommendation for an antibiotic prophylaxis in SCI patients undergoing UDS. However, the EAUguidelines on Urological Infections (http://www.uroweb.org/gls/pdf/ 17_Urological\%20infections_LR\%20II.pdf) suggest considering an antibiotic prophylaxis in UDS 'in high-risk patients' and 'previous urogenital infection' is considered to be one of the most important risk factors. In this respect, as almost all patients have had previous infections, a recommendation for an antibiotic prophylaxis would be derived.

\section{'What ought we to do?'}

On the basis of our results, one should consider to approve the administration of antibiotics for SCI patients with SBU at the time of urodynamics. But because one could not be sure whether a SCI patient presents with sterile urine or SBU to a urodynamic 
examination and the time needed to provide cultural evidence is approximately $24 \mathrm{~h}$, in this regard we are dependent on the use of exploratory detection methods (for example, dipstick test for nitrite and leukocytes esterase).

SCI patients with triggered reflex voiding revealed a high post-UDS UTI-rate in our study, but the number of patients included $(n=19)$ was too low to come to an informed decision about the indication of prophylactic antibiotics.

Otherwise, according to our data the post-urodynamic de-novoinfection rate of SCI patients with IC and of those with sterile urine prior UDS was as low as a general recommendation of antimicrobial prophylaxis may not be justified in these groups.

Summarized, it could not be advisable to advocate antibiotic prophylaxis for all SCI patients due to the incremental threat of bacterial resistance. For this reason the use of antibiotics should be restricted in the challenging population of SCI patients. A widespread application of antibiotics, also with a prophylactic intention, could be more hazardous than beneficial.

\section{'For what may we hope?'}

Our results have to be confirmed or refused by further studies. Wellconducted analysis with appropriate power and a commonly accepted definition of UTI and SBU are urgently needed ${ }^{16}$ to bring up more lucidity in this important topic. Further research has to define patientsubgroups that are on elevated risk for UTI caused by UDS, as well as the onset and the length of prophylactic antibiotic administration. At least different antibiotics have to be evaluated in a risk-benefit analysis.

It would be desirable to establish common standards for all SCI centers to unify the different strategies in the use of antibiotics in SCI patients. ${ }^{17}$

\section{CONFLICT OF INTEREST}

The authors declare no conflict of interest.
1 Watanabe T, Rivas DA, Chancellor MB. Urodynamics of spinal cord injury. Urol Clin North Am 1996; 23: 459-473.

2 Ryu KH, Kim YB, Yang SO, Lee JK, Jung TY. Results of urine culture and antimicrobial sensitivity tests according to the voiding method over 10 years in patients with spinal cord injury. Korean J Urol 2011; 52: 345-349.

3 Singh R, Rohilla RK, Sangwan K, Siwach R, Magu NK, Sangwan SS. Bladder management methods and urological complications in spinal cord injury patients. Indian J Orthop 2011; 45: 141-147.

4 Quek P, Tay LH. Morbidity and significant bacteriuria after urodynamic studies. Ann Acad Med Singapore 2004; 33: 754-757.

5 Manual Neuro-Urology and Spinal Cord Lesion. Guidelines for urological care of spinal cord injury patients. Revised 2007. www.dmgp.at/downloads/leitlinien_urologie.pdf.

6 Schäfer W, Abrams P, Liao L, Mattiasson A, Pesce F, Spangberg A et al. International Continence Society. Good urodynamic practices: uroflowmetry, filling cystometry, and pressure-flow studies. Neurourol Urodyn 2002; 2: 261-274.

7 Wyndaele JJ, Kovindha A, Madersbacher H, Radziszewski P, Ruffion A, Schurch B et al. In: Abrams P, Cardozo L, Khoury S and Wein A (eds). Incontinence. The 4th International Consultation on Incontinence, 10: Neurologic Urinary and Faecal Incontinence ; 5-8 July 2008; Committee, Paris 2009, pp 804-806. ISBN 0-9546956-8-2.

8 Bootsma AM, Laguna Pes MP, Geerlings SE, Goossens A. Antibiotic prophylaxis in urologic procedures: a systematic review. Eur Urol 2008; 54: 1270-1286.

9 Latthe PM, Foon R, Toozs-Hobson P. Prophylactic antibiotics in urodynamics: a systematic review of effectiveness and safety. Neurourol Urodyn 2008; 27: 167-173.

10 Lowder JL, Burrows LJ, Howden NL, Weber AM. Prophylactic antibiotics after urodynamics in women: a decision analysis. Int Urogynecol J Pelvic Floor Dysfunct 2007; 18: 159-164.

11 Joseph AC, Albo M. Urodynamics: the incidence of urinary tract infection and autonomic dysreflexia in a challenging population. Urol Nurs 2004; 24: 390-393.

12 Darouiche RO, Smith MS, Markowski J. Antibiotic prophylaxis for urodynamic testing in patients with spinal cord injury: a preliminary study. J Hosp Infect 1994; 28: 57-61.

13 Pannek J, Nehiba M. Morbidity of urodynamic testing in patients with spinal cord injury: is antibiotic prophylaxis necessary? Spinal Cord 2007; 45: 771-774.

14 Esclarín De Ruz A, García LE, Herruzo CR. Epidemiology and risk factors for urinary tract infection in patients with spinal cord injury. J Urol 2000; 164: 1285-1289.

15 Consortium for Spinal Cord Medicine. Bladder Management for Adults with Spinal Cord Injury: a Clinical Practice Guideline for Health Care Providers. Paralyzed Veterans of America: Washington (DC), 2006, 50 pp. www.metrohealth.org/documents/patient\% 20services/norscis/Bladder.pdf.

16 Wyndaele JJ, Bruschini H, Madersbacher H, Moore K, Pontari M, Wein A. Neurological patients need evidence-based urological care. Neurourol Urodyn 2010; 29: 662-669.

17 Pannek J. Treatment of urinary tract infection in persons with spinal cord injury: guidelines, evidence, and clinical practice. A questionnaire-based survey and review of the literature. J Spinal Cord Med 2011; 34: 11-15. 